\title{
Lattices align in two dimensions
}

Science 359, 1131-1136 (2018)

Semiconducting two-dimensional materials, such as transition-metal dichalcogenides, are a promising alternative to graphene for future electronic devices. However, building high-quality heterojunctions, which would be required for particular devices, from lattice-mismatched 2D layers can be challenging, since conventional epitaxy can introduce dislocations or defects into the films. Jiwoong Park and colleagues at Cornell University and the University of Chicago have now grown monolayer superlattices in which different transition-metal dichalcogenides $-\mathrm{WS}_{2}$ and $\mathrm{WSe}_{2}$ - are coherently integrated, despite a lattice mismatch of $4 \%$.

The researchers developed an omnidirectional epitaxial technique that forms superlattices along the growth plane. The process is a modified chemical vapour deposition method that offers precise strain control of the monolayers. In conventional unidirectional epitaxy, the final structure can be asymmetric because layers with different lattice constants are deposited one after the other. In contrast, with this new approach layers with different epitaxial directions adapt and connect to each other during growth, maintaining symmetry within the crystal. As a result, the superlattices display uniform lattice constants and minimal dislocations, which was verified by electron microscopy imaging.

As a further assessment of the approach, Park and colleagues used the heterostructures to create transistors and light-emitting diodes that exhibited high rectification ratios.

Christiana Varnava

Published online: 17 April 2018

https://doi.org/10.1038/s41928-018-0065-5 